\title{
Trojan Microparticles Potential for Ophthalmic Drug Delivery
}

\author{
Sergio Esteban Pérez, Irene Bravo-Osuna*, Vanessa Andrés-Guerrero, Irene T. Molina-Martínez, Rocío \\ Herrero-Vanrell \\ Complutense University, InnOftal Research Group, UCM 920415, Department of Pharmaceutics and \\ Food Technology, Faculty of Pharmacy, Plaza Ramón y Cajal s/n, Madrid, 28040, Spain. Ocular \\ Pathology National Net (OFTARED) of the Institute of Health Carlos III, Spain. Health Research \\ Institute of the San Carlos Clinical Hospital (IdISSC), Madrid, Spain
}

*Corresponding author: ibravo@ucm.es; Phone: +34 91394 1737; Fax: +34 913941736

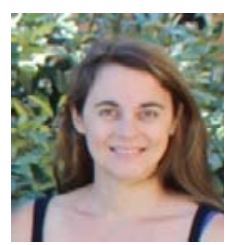

\begin{abstract}
The administration of drugs to treat ocular disorders still remains a technological challenge in this XXI century. Although there is an important arsenal of active molecules useful to treat ocular diseases, ranging from classical compounds to biotechnological products, there is not currently any ideal delivery system able to profit all their therapeutic potential. Among the intraocular drug delivery systems (IODDS) proposed to overcome some of the most important limitations, microsystems and nanosystems have raised high attention. While microsystems are able to offer long-term release after intravitreal injection, nanosystems can protect the active compound from external environment (reducing their clearance) and direct it to its target tissues. In recent years, some researchers have explored the possibility of combining micro and nanosystems in "nanoparticle-in-microparticle (NiMs)" systems or "trojan systems". This excellent idea is not exempt of technological problems, that remains partially unsolved, especially in the case of IODDS. The objective of the present review is to show the state of art concerning the design, preparation and characterization of trojan microparticles for drug delivery and to remark their potential and limitations as IODDS, one of the most important challenge faced by pharmaceutical technology at the moment.
\end{abstract}

Keywords: Trojan systems, nanoparticles-in-microparticles (MiMs), ophthalmic drug delivery, posterior segment, intravitreal injection, intraocular drug delivery systems (IODDS).

\section{INTRODUCTION}

The administration of drugs to treat ocular disorders still remains a technological challenge in this XXI century. Although there is an important arsenal of active molecules useful to treat ocular diseases, ranging from classical compounds to biotechnological products, there is not currently any ideal delivery system able to profit all their therapeutic potential.

When the target tissue is located in the anterior segment of the eye the ocular topical route is preferred. This local administration results non-invasive and relatively comfortable for patients. However, most of the active compounds have low local bioavailability due to the reduced contact time on the ocular surface of traditional eye drops and to the low inherent corneal permeability of most of the active compounds.

The challenge is still bigger when the target tissue is in the back of the eye. Periocular routes, peribulbar, retrobulbar, posterior yuxtascleral, subtenon or subconjunctival routes (Figure 1), are interesting options. The formulation is injected in different zones surrounding the eye globe however the drug has to be able to reach itself the intraocular tissues by diffusing through the sclera, the choroid and, depending on the injection site, also though the aqueous humor and vitreous [1, 2].Intraocular administration routes involve the direct deposit of the formulation in the proximity of the target tissue into the ocular globe, to treat pathologies affecting the anterior segment of the eye (intracameral route) [3], and also the posterior segment of the eye (intravitreal route, subretinal route, etc.) [4] (Figure 1).

Figure 1. Schematic representation of eye and ocular administration routes.

\footnotetext{
**Corresponding author: Irene Bravo Osuna Phone: +34 91394 1737; Fax: +3491394 1736; E-mails: ibravo@ucm.es
} 
Intravitreal injections are preferred in clinic to treat vitreoretinal diseases. However, several issues limit their utility. When the whole dose is locally deposited, the high initial drug concentration achieved might result toxic for the retina. However, after that, the vitreous concentration of drugs rapidly drops to low values due to different clearance mechanisms. Although the elimination of active compounds from the vitreous depends on several factors such as size and polarity of the molecule, in many cases half-life values of only several hours are found [5]. All these events joined to the fact that most part of vitreoretinal disorders are chronic diseases, make necessary the use of repeated intravitreal injections for a successful therapy. Unfortunately, repeated intravitreal injections are associated to important adverse effects such as cataracts, retinal detachment, haemorraghes, risk of infections, among others, due to the injection itself.

For more information concerning the above-mentioned information, researchers are kindly suggested to read several recent complete reviews about the subject [2, 6-8].

In the case of diseases affecting the posterior segment of the eye, several kinds of devices such as implants $(>1 \mathrm{~mm})$ and microsystems $(1-1000 \mu \mathrm{m})$ have been proposed. These intraocular drug delivery systems (IODDS) are able to provide sustained delivery of the loaded active compound in the vicinity of the target site for long periods of time. In fact, several implants are currently used in the clinical practice with high acceptance from patients and professionals. Concerning to clinical use, microparticulate systems have been less explored than implants but offer important advantages. For example, they can be injected using the conventional needles employed for intraocular administration. Depending on the biomaterial used in their preparation, microparticles can agglomerate once administered, forming an in situ depot, able to release the active compound for months. If biodegradable materials are employed, the IODDS gradually disappears from the injection site once released the active compound. Furthermore, as the amount of administered microparticles can be easily modified, they are very suitable for personalized therapies $[6,7]$. Several authors have demonstrated the usefulness of microparticles for effective delivery of active compounds after intravitreal administration [9-11]

Nanosystems have been also proposed as IODDS. Due to their small size $(1-1000 \mathrm{~nm})$ and to the possibility of presenting different surface nature, they can be designed to undergo cell internalization. Furthermore, they can protect the active compound form vitreal environment. Numerous studies have demonstrated the utility of nanosystems for the effective administration of active compounds at retinal level [12-16].

According to the previously exposed, on one hand, microsystems are able to offer long-release after intravitreal injection, and on the other hand, nanosystems can protect the active compound from external environment, reducing their clearance, and directing it to its target tissue. In recent years, some researchers have explored the possibility of combining micro and nanosystems to create a new generation of drug delivery systems with the potential advantages of both. These are the named "nanoparticle-in-microparticle (NiMs) systems or, more poetically, "trojan systems".

The trojan systems can be then defined as microparticles that are loaded with nanoparticles, which, in turns, carry the active compound. Ideally, once administered, the microparticles will slowly release the nanoparticles for long periods, protecting the unreleased nanoparticles from external environment. Once released, nanoparticles will be then directed to the target cells, internalized and will release the active compound into the cell (figure 2). This would allow maximizing the efficacy of the administered active molecule, by minimizing their clearance before reaching the target cell.

Figure 2. Schematic representation of trojan microparticles delivering nanoparticles close to the retinal and further nanoparticles cell internalization.

This excellent idea is not exempt of technological problems that remains partially unsolved at present, such as the efficient encapsulation of nanosystems in microparticles, the achievement of reproducible microparticle size, release patterns of nanosystems from microparticles and of active compounds from nanoparticles, etc. It is important to take in mind that all this must be achieved by using mild preparation techniques in order to preserve the active compound integrity, especially if it is a bioengineered compound. Furthermore, in the special case of IODDS additional limitations such as the use of only extremely well tolerated biomaterials for the preparation of the trojan systems and also the need of sterilization, makes the design, preparation and characterization of trojan systems for intraocular drug delivery one of the most important challenge faced by pharmaceutical technology at the moment.

The objective of the present review is to show the state of art concerning the design, preparation and characterization of trojan microparticles (nanoparticles-in-microparticles, NiMs 
systems) for drug delivery and to remark their potential and limitations as IODDS.

\section{TROJAN MICROPARTICLES. TECNOLOGICAL APPROACH}

\subsection{Trojan microparticles composition and elaboration}

Several techniques of microencapsulation have been adapted to allow the effective entrapment of nanoparticles. In this part, the main microencapsulation methods employed to prepare trojan systems will be mentioned.

\subsubsection{Emulsion techniques}

Some of the techniques most commonly used to prepare trojan microparticles are based on the formation of an emulsion and subsequently solvent extraction/evaporation. Amiji and collaborators published between 2006 and 2011 several works showing the benefit of NiMs systems of gelatin nanoparticles in poly-caprolactone microparticles for oral gene delivery [17-20]. These were some of the pioneer works showing the potential benefit of the micro-nano combination. They first prepared gelatin nanoparticles by desolvation and controlled precipitation using ethanol as non-solvent. After preparation, nanoparticles were lyophilized and subsequently suspended in water, forming the inner aqueous phase of a $\mathrm{W} / \mathrm{O} / \mathrm{W}$ double emulsion to prepare the final poly-caprolactone microparticles. Authors identified three critical parameters affecting the trojan system particle size: concentration of policaprolactone in the O-phase, speed of the homogenization to form the $\mathrm{W} / \mathrm{O} / \mathrm{W}$ emulsion and, importantly, the amount of nanoparticles included in the inner $\mathrm{W}$ phase of the double emulsion. According to the authors, when the amount of nanoparticles in the inner aqueous phase increases, the collision and fusion of droplets of this aqueous phase is reduced during the primary $\mathrm{W} / \mathrm{O}$ emulsion, leading to smaller microparticles after the $\mathrm{W} / \mathrm{O} / \mathrm{W}$ emulsion [19]. Years later, Lee et al (2013) [21] performed a deep analysis of the different technological variables affecting the preparation of trojan microparticles by $\mathrm{W} / \mathrm{O} / \mathrm{W}$ emulsion. They firstly prepared nanoparticles using polycaprolactone as polymer entrapping the drug hidrocortisone. Then, these nanoparticles were included as solids in the inner aqueous phase of the double emulsion and prepared PLGA microparticles, forming a $\mathrm{S} / \mathrm{W} / \mathrm{O} / \mathrm{W}$ system. These authors paid especial attention on the state of nanoparticles, evaluating their incorporation as dry powder but also as "slurry mass". According to the results previously described, these authors suggested that the distribution of nanoparticles within the primary emulsion influences their ultimate destination in the final microparticle. In this study they demonstrated that the inclusion of nanoparticles with high tendency to agglomerate ("slurry mass") led to better internalization in microparticle structure, while dry nanoparticles presented more tendency to locate in the vicinity of microparticle surface and even on the microparticle surface. This different nanoparticles distribution will have a strong influence not only on the encapsulation efficiency but also on the release profile. Chen et al., (2014) [22] also used the $\mathrm{S} / \mathrm{W} / \mathrm{O} / \mathrm{W}$ method to prepare PLGA brucine-loaded nanoparticles dispersed in PLGA microparticles with the aim to overcome the problem of burst release and to prolong the release time.

Single emulsion has been also used to prepare trojan microparticles. For example, Farris et al., (2017) [23] initially prepared chitosan nanoparticles including DNA plasmid by ion gelation. Nanoparticles were suspended in distilled water and the suspension was mixed with a solution of zein in ethanol. This hydro-alcoholic media was emulsified with an oily external phase (corn oil). After solvents evaporation zein microparticles encapsulating chitosan/plasmid nanoparticles were recovered.

Additional novel microencapsulation methods based on emulsions formations are also being evaluated to prepare trojan systems. For example, Khan et al (2015) [24] used a microfluidic system to prepare trojan nanoparticles in two semi-continuous steps. They first prepared an $\mathrm{O} / \mathrm{W}$ nanoemulsion containing the active compound (ketoprofen) and acrylic monomers. This nanoemulsion was subequently emulsified with an aqueous phase containing acrylamide monomers and different surfactants. The microdroplets (and the nanoemulsion included in them) were hardened by UVinduced polymerization of the corresponding monomers. Guo et al., (2014) [25] prepared calcium phosphate nanoparticles loaded with a plasmid encoding two growth factors. The nanoparticles were included in microparticles prepared by electrospray using a mixture of poly(ethylene glycol) (PEG) blended with the copolymer poly(ethylene glycol)-poly(Lactide) (PELA). According to authors, electrospray techniques offer a low-cost and rapid method to obtain particles with high production efficiency. The method employed was based on the fact that a strong electric field during electro-spraying can break up a jet liquid into a continuous stream of finely dispersed droplets. To obtain the trojan systems the liquid used was a previously formed emulsion formed by an inner aqueous phase of nanoparticles suspended in distilled water and an external phase of the polymer solubilized in an organic solvent (dimethylformide). After the droplet formation, the organic solvent was evaporated during flying to the collector. The microparticles created were collected in water, centrifuged and finally freeze-dried.

\subsubsection{Spray drying techniques}

Spray drying is another of the technological approaches evaluated to produce trojan microparticles. For example, Elbaz et al (2016) [26] prepared several nanoparticulate systems containing propolis extract (solid dispersion, pluronic nanoparticles and PLGA nanoparticles) within chitosan microparticles. The method employed was spraydrying of a quitosan aqueous solution containing the nanoparticles suspension. The idea of authors is that this nanoparticles-in-microparticles structure might be able not only to control the release of the active and protect it from gastrointestinal environment after oral administration, but also to increase the propolis extract aqueous solubility.

Ozeki et al., (2012) [27] developed a spray-drying method able to prepare in only one-step nanoparticles in 
microparticles. To this, authors used a two-solution mixing type nozzle, which is able to mix an organic media containing components necessary to create the nanoparticles and an aqueous media including the microparticles forming polymers. This method has several relevant advantages. It reduces the manipulation of nanoparticles as intermediate products and also interestingly it allows the preparation of the trojan systems without any solvent or surfactant. At the moment this technique has been used to prepare nanoparticulate pure drugs or empty polymeric nanoparticles (ethylcellulose), both in mannitol microparticles. Although none of these prototypes can be considered "trojan systems" as described in this review, this technique could be used in the future to prepare them.

Although, as mentioned before, in most of the cases trojan microparticles are being explored to protect the active compound from external environment and to prolong its release, some alternative interesting strategies focused on targeting have been also proposed. For example, Deng et al., (2014) [28] prepared cationic mesoporous silica nanoparticles loaded by electrostatic interaction with ovoalbumin (as model antigen). The nanoparticles were then included in an aqueous solution of different sugars (trehalose, mannitol) and dextran that was finally freeze dried to obtain the microparticles. In this case the objective was not to extend the delivery of the active compound, but to create microcarriers able to meet all physical parameters required for intradermal powder injection (particle size big enough to have good flowability and to reduce the risk of clumping in the syringe). Once injected, the sugar-based microparticles dissolves in the subcutaneous media, rapidly releasing all nanoparticles in a high concentrated sugar matrix, which in turns offers additional protection to the released protein. Tewes et al., (2014) [29] used a spraydrying method to prepare polyethylene glycol (PEG)/hydroxypropyl-beta-cyclodextrin and ammonium carbonate microparticles containing superparamagnetic iron oxide nanoparticles. The rational of this design was the preparation of particles able to reach deep lung alveolar regions with the external direction of a magnetic field, avoiding all aggregation typically occurring to nanoparticles alone after inhalation. The microparticles size was optimized by modifying the atomization procedure to reach the $1-5 \mu \mathrm{m}$ range. Unfortunately, no drug loading was performed in the work, however, authors claimed that if the nanoparticles were loaded with active compounds the trojan systems generated could be very useful in the local treatment of lung diseases such as cancer nodules or bacterial infectious foci. In this same line, Bakhtiary et al., (2017) [30] also used microparticles with optimal size, flowability and aerodynamic behavior, able to reach the desired part of lung after inhalation. Once deposited, microparticles, made of mannitol by spray-drying, rapidly released the solid lipid nanoparticles embodied which were loaded with an anticancer drug (erlotinib). Schuze et al., (2018)[31] also used the spray drying technique to prepare a nanoparticlesin-microparticles inhalatory gene delivery system. The trojan systems were generated by combination of polyethyleneimine (PEI) based polyplexes and lipopolyplexes into polyvinyl alcohol (PVA) microparticles.
The previously prepared nanosystems were suspended in PVA solution and directly spray-dried without any stabilizing agent. Microparticles resulted in the 3-5 $\mu \mathrm{m}$ range and spherical. Due to the hydrophilic nature of the polymer employed to prepare the microparticles, once in contact with aqueous media the trojan system rapidly disintegrates releasing the nanosystems.

\subsubsection{Gelation techniques}

In an interesting work, Pinkerton et al., (2013) [32] prepared trojan microparticles by gelation. Authors first produced polystyrene nanoparticles that were then included in polyethylene glycol (PEG) microgels obtained by different crosslinking methods. This last step was achieved by inclusion of nanoparticles in a PEG solution that was then emulsified with silicon oil. UV radical polymerization and Michael addition polymerization reaction were proposed as possible techniques to obtain the final microgels. This further was the selected, so no reduction of fluorescence was obtained when a fluorescent marker was included in nanoparticles, demonstrating that Michael reaction maintained the integrity of the encapsulated compound, while UV exposure promoted the degradation of encapsulated compound by radical attack. Particles were prepared in a range size suitable for effective lung targeting (drug delivery and imaging) after intravenous administration via venous filtration pathway. Once intravenously injected microparticles are trapped in lung tissues the hydrogel is progressively degraded to release the nanoparticles. In a different approach, ionic gelation was used by Garrati et al (2014) [33] to prepare alginate microparticles containing chitosan nanoparticles. Nanoparticles were prepared by precipitation/coacervation method containing amaranth red as model loaded molecule. Chitosan nanoparticles were included in an alginate solution that was forced to pass trough a vibrating nozzle to create microdrops. These microdrops were collected in a calcium chloride solution to promote the ionic crosslinking of alginate forming then the microparticles. Trojan microparticles were finally spraydried. Authors considered as main critical technological point the preparation of an homogeneous suspension of nanoparticles in alginate solution and also the nanoparticles/alginate ratio. It was observed that when high concentrations on nanoparticles were employed immediate precipitation occurred, so only nanoparticles suspension bellow $0.2 \%$ resulted adequate. Subsequently, authors also demonstrated that a high alginate concentration was necessary to obtain spherical trojan nanoparticles. Interestingly, special attention was also paid in minimizing the "drug" release from nanoparticles in the alginate solution. To this, several parameters such as adjusting $\mathrm{pH}$ and including a non-ionic surfactant (Tween 80) resulted useful. Effectively, depending on the microencapsulation procedure used to prepare the trojan systems and on the physicochemical characteristics of the active compound, in some cases the release of loaded active compound from the nanoparticles during the microencapsulation process must be considered.

\subsection{Trojan microparticles characterization}


Some techniques generally used to describe simple microparticles can be also used in the case of trojan microparticles, such as main particle size and particle size distribution measurements by dynamic light scattering techniques or microscopy, surface morphology evaluation by scanning electronic microscopy, etc. However other techniques such as quantification of loaded active compound in the trojan system or in vitro release profiles studies need to be modified in order to better characterize the new systems. Furthermore, additional techniques rarely employed for microparticles characterization became relevant in the case of trojan systems.

\subsubsection{Active compound encapsulation efficiency in Trojan microparticles}

The quantification of the active compound loaded in the trojan systems generally must be carried out in several steps. In the first one the nanoparticles need to be extracted from the polymeric matrix forming the microparticle. The most used method to aim this goal is to dissolve the trojan microparticles in a solvent in which nanoparticles are insoluble. After that, the recovery of nanoparticles is performed by extraction with a suitable solvent. Finally, the media containing the extracted nanoparticles has to be treated to separate and quantify the active compound. All these steps can compromise the stability of the active compound, especially if it is a bioengineered product (oligonucleotides, peptides, proteins), so bioactivity studies are required at the end of the study. Amiji and co-workers used a combination of methylene chloride and distilled water to dissolve polycaprolactone, polymer used to prepare the trojan microparticles, and extract the embodied gelatin nanoparticles, respectively. Further treatment consisting on dissolving gelatin by using proteases and subsequent fluorescent quantitation assays were performed to determine the amount of plasmid DNA initially loaded in the trojan microparticles [17]. In the same sense, Guo et al., (2014) [25] dissolved the PEG-PELA trojan microparticles in methylene chloride. The calcium phosphate nanoparticles included in them were extracted several times in aqueous media. After that, the plasmid DNA loaded in nanoparticles was quantified by fluorescence measurement.

In some cases it is also possible to direct degrade microparticles and loaded nanoparticles in the same step. For example, Garrati et al (2014) [33] disrupted their whole trojan systems (alginate microparticles containing chitosan nanoparticles) by dissolving them in a sodium bicarbonate and tri-basic sodium acetate at $\mathrm{pH} 8$.

Other authors have opted to perform more simple methods. For example, Khan et al., (2015) [24] simply incubated the trojan microparticles in release media for 48 hours to, according to authors, complete extract the active compound (ketoprofen) included in the trojan systems (composed by nanoparticles prepared with acrylic polymers and then microencapsulated in acrylamide polymer). However, additional studies demonstrated that part of the drug could not be extracted at these conditions. So, in general, it does not seem an appropriated method to determine the active compound encapsulation efficiency.
When the active compound is radiolabeled, direct measurement can obviously be performed [23] without need of extraction procedures.

Some authors only evaluate the drug loading efficiency on initial nanoparticles without any further evaluation of the trojan system in this sense, assuming that no waist of material nor drug degradation occur during the microencapsulation procedure $[26,30]$. However, as general rule, these encapsulation efficiency data might be taking into account only as approximated values.

\subsubsection{In vitro release profile of the loaded active compound from trojan microparticle}

When performing in vitro release studies from classical microparticles, typically a known amount of them are placed in a tube filled with release media at controlled temperature and shaking. Supernatant samples are periodically extracted and measured to determine the concentration of released active compound. However, when trojan systems are used, the study of the release profiles becomes more complicated.

On one hand, some authors simply evaluate the amount of active compound released form the trojan system, with some modifications from the basic methodology. For example, Amiji and co-workers used a release media containing proteases to ensure nanoparticles (prepared with gelatin) dissolution once released, and the plasmid DNA incorporated in them was directly measured in the supernatant by fluorescence measurements [17]. Using this methodology both, the nude active compound released from the trojan systems and the active compound included in the released nanoparticles are jointly quantified. Other authors such as Khan et al., (2005), Chen et al (2014) or Elbaz et al (2016) $[22,24,26]$ used dialysis bags or dialysis tubes to separate the trojan system and nanoparticles delivered from the released active compound that can be then easily quantified.

However, a more complete characterization of the trojan system can be achieved if, as previous steps, the method is modified to determine the amount of nanoparticles released from the trojan system. With this aim, several authors have explored some astute alternatives. For example, Khan et al (2015) [24] incubated the trojan system in release media for two hours and then they observed the supernatant under transmission electronic microscopy (TEM) to determine the presence of nanoparticles. No quantification studies were able with this technique. In this sense, Guo et al (2014) [25] evaluated the in vitro plasmid DNA release from the Trojan nanosystems created (calcium phosphate nanoparticles in PEG-PELA microparticles) in two parallel studies. In one of them microparticles were incubated in release media, the supernatant was taken at fixed time points, and the released nanoparticles were determined by electrophoresis. In a second set of experiments the release media was used to quantify the total released plasmid DNA.

2.2.3. Imagine techniques and other methods useful for trojan microparticles characterization 
Since it is so easy to prepare nanoparticles including fluorescent markers, the use of fluorescent microscopy (and even better confocal microscopy) becomes a very useful tool to determine the distribution of nanoparticles into the polymeric microparticle. $[21,24,28]$. The direct visualization of loaded compounds into the polymeric matrix has been sometimes used to characterize classical microparticles [10]. This technique offers very useful information. It confirms the trojan morphology and it can result a very interesting tool during the optimization of the microencapsulation process. Additionally, it allows the prediction of the in vivo behavior (whether nanoparticles will remain in the polymeric matrix structure or will rapidly scape from it). This technique was used by Lee et al., (2013) [21] to observe the different distribution of polycaprolactone nanoparticles in PLGA microparticles when they were incorporated as dry powder in a $\mathrm{S} / \mathrm{W} / \mathrm{O} / \mathrm{W}$ emulsion or as "slurry mass". The confocal observation of trojan systems is even more informative when it is possible to simultaneously observe both, the nanoparticles and the microparticle matrix. With this idea, Garrati et al (2014) [33] used two label molecules to identify chitosan nanoparticles (fluorescein) inside alginate microparticles (rhodamine). Using this technique authors were able to observe a homogeneous distribution of nanoparticles within the microparticle polymeric matrix.

The distribution of nanoparticles within the trojan microparticles can be also evaluated by scanning electronic microscopy (SEM) evaluation of cryo-fractured microparticles [21, 24]

Bhavsar et al., 2007 [17] used SEM studies to determine whether the trojan systems created (poylcaprolactone microparticles included gelatin nanoparticles entrapping plasmid DNA) were able to protect the nanoparticles from external environment. They incubated the trojan systems in a media including proteases (able to digest gelatin) and observed no degradation of the system after a $2 \mathrm{~h}$-incubation. Incubation of trojan microparticles in different media mimicking biological conditions can also result very useful. For example, Farris et al., (2017) [23] prepared chitosan nanoparticles included in zein microparticles. Zein is a corn protein able to protect nanoparticles for stomach media after oral administration. To confirm that, they incubated the microparticles at $\mathrm{pH} 1.2$ in a media containing pepsin for 15 min a $37^{\circ} \mathrm{C}$ and after that they evaluated the transfection efficiency of the system in comparison with untreated samples. They observed the same transfection capacity in both cases demonstrating the total protection of nanoparticles from external environment. Similarly, Guo et al., (2014) [25] demonstrated the protective effect of the trojan system created (calcium phosphate nanoparticles loaded with plasmid DNA and included in PEG-PELA microparticles) by quantification of plasmid DNA after subjecting them to DNase I digestion for 48 hours.

\section{TROJAN MICROPARTICLES POTENTIAL FOR THE TREATMENT OF OCULAR DISEASES.}

As it has been pointed out in the text, although nanoparticles alone offer interesting advantages in targeting and cell internalization, for some administration routes microparticles are preferred. As mentioned before, for example in the case of transdermal administration, pulmonary administration or venous filtration pathway to the lung. In these cases nanoparticles alone are too small to reach the target tissue. They can agglomerate in the injection site, they cannot be deposited in the desired part of the affected organ or they cannot be retained by filtration. In general, nanoparticles are difficult to manipulate, not only in its dry form but also as suspension, so they can undergo hydrolysis, sedimentation, etc. [34].

When treating diseases affecting the back of the eye the size of particles also strongly influences their behavior and location after injection. Microparticles generally tend to deposit (aggregated or not, depending on the nature of the biopolymer used) not perturbing the patients' vision and are retained in the ocular cavity. Only in absence of crystalline they have been located in the anterior chamber [35]. On the contrary, when a nanoparticles suspension is injected in the vitreous their distribution seems to strongly depend on the surface characteristics [36]. For example, cationic nanosystems (those more promising for gene delivery) can be retained in the vitreous by electrostatic interaction with anionic intravitreal collagen fibrins dispersed in the whole cavity. On the contrary, anionic nanoparticles can be cleared from the vitreous diffusing to retinal layers and other ocular tissues [37]. Although the toxicity of nanoparticles in the vitreous is not well evaluated in the scientific literature, it seems that a massive presence of nanoparticles in the vitreous could activate hyalocytes, the sentinel immune cells of the vitreous, depending on the nanoparticles chemical nature [38]. Some studies have demonstrated that the size of particles can affect also their distribution after periocular administration. For example, Amrite et al (2005) [39] observed that while microparticles could be retained in the site of injection after subconjunctival injection for several weeks, nanoparticles (20 and $200 \mathrm{~nm})$ tend to be rapidly cleared from subconjunctival space after injection due to systemic and lymphatic circulation.

The combination of nano- and microsystems in trojan microparticles for intraocular delivery might offer several benefits. On one hand the facility of manipulation and the more controlled behavior once injected in the vitreous, characteristics of microparticles, in addition to the tuning of sustained release for long periods. On the other hand, the embodied nanoparticles would offer all the advantages in terms of active compound protection and cell internalization.

Gómez-Gaete et al (2008) [34] developed an interesting work proposing PLGA (75:25) nanoparticles loaded with dexamethasone acetate included in microparticles composed by a combination of a phospholipid (1,2-Dipalmitoil-sn-Glycerol3-phosphocholine -DPPC-) with hyaluronic acid (HA). Dexamethasone is a corticoid anti-inflammatory drug useful in the treatment of several posterior segment eye diseases, such as uveitis, proliferative vitreoretinopathy or subretinal neovascularization among others [40]. The use of IODDS is being explored to reduce the number of applications and the side effects associated to massive presence of the corticoid in 
the eye such as cataracts. Authors prepared dexamethasone loaded PLGA nanoparticles by an $\mathrm{O} / \mathrm{W}$ solvent emulsionevaporation technique. After hardening of nanoparticles, separation of dexamethasone crystals by filtration and from soluble dexamethasone by ultacentrifugation, fresh (non freezedried) nanoparticles were suspended in water. In parallel, DPPC/HA hydro-alcoholic solution was prepared. After that, the polymer solution and the nanoparticle suspension were pumped using a two digital gear pump connected to an acetal helix that performed the mixture on the two media before spray drying. As mentioned before, it is necessary to guarantee that the loaded drug remains in the nanoparticles during the microparticles preparation. That is why authors decided to separately pump the nanoparticle suspension and the hydroalcoholic solution and then mix the two liquids just before the spray, reducing the contact time between nanoparticles and ethanol that might promote the partial solution of dexamethasone. The final trojan systems created were characterized using some of the techniques already exposed before. Different concentrations of nanoparticles (2.2-3 g/L) during the freeze-drying step were tested to optimize the trojan microparticles mean particle size. Analysis of the different batches prepared showed non-substantial changes on particle mean size and particle size distribution, with values around 1 $\mu \mathrm{m}$ for $\mathrm{D}_{10}$ and $16 \mu \mathrm{m}$ for $\mathrm{D}_{90}$, neither on the production yield (around 33\%). Similarly, SEM and confocal observations demonstrated that the so prepared microparticles resulted in a hollow shell structure, both in absence and in presence of nanoparticles. In previous studies authors demonstrated that hyaluronic acid was the responsible for this conformation. Interestingly, the presence of nanoparticles influenced the microparticles surface, changing from a smooth aspect to a roughness one, due to the clear presence of nanoparticles on it. Confocal images also demonstrated that nanoparticles resulted homogenously distributed within the polymeric matrix and in general remained as individual entities, no matters the concentration used during the microparticles formation. However exhaustive studies of the nanoparticle size after spraydrying showed a significant increment in the mean size values of nanoparticles (from $200 \mathrm{~nm}$ to $230 \mathrm{~nm}$ ) and a broad particle size distribution, indicating some extent of nanoparticle irreversible aggregation during microparticles formation. The inclusion of dexamethasone-loaded nanoparticles in the trojan systems promoted a change in its in vitro release. When free nanoparticles were tested the release of the $90 \%$ of dexamethasone release from them was observed in the first 4 hours and the remained $10 \%$ was released before 72 hours. For trojan systems a more sustained and prolonged release was obtained with a $80 \%$ of drug content released after $72 \mathrm{~h}$ of in vitro study. However, in the case of trojan systems, interestingly a more important burst effect was observed when compared with free nanoparticles dexamethasone in vitro release, in spite of the higher specific surface offered by unprotected nanoparticles. This fact was explained as consequence of partial leakage of the active compound from nanoparticles to microparticles matrix during trojan system formation, demonstrating that even having all the possible cautions, this fact has to be always taken into account. Unfortunately authors have not published at the moment any in vivo study to confirm the real potential of the interesting trojan systems proposed, however, they claimed that "the in situ release of drug loaded nanoparticles should favor their internalization within retinal pigmented epithelial cells and might therefore increase the drug efficacy".

Bevacizumab in a full-length antibody with anti-VEGF (vascular endothelial growth factor) activity. It was originally developed for the treatment of several cancer diseases (colorectal, lung or brain) but it is also used in ophthalmology to treat neovascularization retinal diseases such as wet age-related macular degeneration (AMD), characterized by an abnormal blood vessel growth in the macula. The current treatment of wet AMD with bevacizumab is based on a intravitreal monthly injection of the protein solution. This is not only extremely uncomfortable and dangerous for patients but also results very expensive. That is why several research groups are currently investigating the use of IODDS to overcome all these limitations [8]. Yandrapu et al (2013) [41] elaborated bevacizumab-loaded nanoparticles using poly lactic acid (PLA) $(1.0 \mathrm{dL} / \mathrm{g})$. As first step, plain PLA nanoparticles were prepared using the $\mathrm{O} / \mathrm{W}$ solvent extraction/evaporation method. After isolation, PLA nanoparticles were subsequently freeze-dried in a bevacizumab solution to promote the protein adsorption onto the nanoparticles surface. The so prepared Bevacizumab-loaded nanoparticles (2.5 mg protein/500 mg PLA; final mean particle size 265 $\mathrm{nm})$ were then included in porous PLGA $(50: 50 ; 0.67 \mathrm{dL} / \mathrm{g}$ ) microparticles elaborated by supercritical infusion and pressure quench technology. To this, plain PLGA microparticles (around $1.6 \mu \mathrm{m}$ ) were prepared also using the $\mathrm{O} / \mathrm{W}$ solvent extraction/evaporation method. Then a mixture of bevacizumab-loaded PLA nanoparticles and PLGA plain microparticles was prepared. The mixture was placed in a high pressure vessel and was exposed to supercritical $\mathrm{CO}_{2}$. At these conditions amorphous PLGA enables the diffusion of $\mathrm{CO}_{2}$ into its polymeric network. Supercritical $\mathrm{CO}_{2}$ acts as a plasticizer able to alter the polymeric network and increase the chain mobility. Upon depressurization $\mathrm{CO}_{2}$ rapidly expands generating pores in the microparticles matrix and increasing its size $\times 7$. As nanoparticles are prepared with crystalline PLA, they do not undergo all this process remaining unaltered, and simply diffuse through the PLGA porous matrix to finally locate inside the microparticle and to form then the final trojan system.

As part of the optimization study, several mixtures of bevacizumab-loaded PLA nanoparticles and PLGA plain microparticles were evaluated at different nano/micro proportions (5/95-25/75). Differences were mainly observed in the in vitro release profile. When high percentage of nanoparticles was include in microparticles $(25 \%)$ an intense burst effect was observed, with a $52 \%$ of total loaded protein released within the first day. After that, a sustained release was observed and the $75 \%$ of the active content was released at day 21 of the release assay. On the contrary, when low proportions of nanoparticles were employed $(5-20 \%)$, the burst effect was progressively reduced to $21-27 \%$ and the in vitro release was extended with $67-81 \%$ of release during 4 months. According to this, the trojan system containing $10 \%$ of nanoparticles and $90 \%$ of microparticles was selected for 
in vivo studies. It is interesting to remark that in this nanoparticle-in-microparticle approach, the use of nanoparticles is not focused on their cell internalization properties neither on their ability to protect the active compound from the external environment, so once the nanoparticle is released from the porous microparticles, the adsorbed protein is rapidly released. The use of nanoparticles in this case simply ensures the progressive release of the compound. In fact, when additional studies were performed including directly a bevacizumab solution, in absence of nanoparticles, to the PLGA microparticles prior to porosification process, the resulting loaded microparticles were unable to efficiently control the protein release, extending it only for 7 days. Several techniques such as size exclusion chromatography, gel electrophoresis, circular dichroism and fluorescence spectroscopy were used to confirm the stability of bevacizumab after microencapsulation and release. SEM pictures allowed the direct observation of nanoparticles inside open PLGA microparticle' porous. This disposition was also confirmed by confocal microscopy observation. To this, authors prepared trojan microparticles using nile red and 6-coumarin to label PLA nanoparticles and PLGA microparticles respectively. In vivo studies were performed in healthy rats by intravitreal injection of $5 \mu \mathrm{L}$ of microparticle suspension $(30 \% \mathrm{w} / \mathrm{v} ; 7.2 \mu \mathrm{g}$ of bevacizumab). Control studies were performed by the injection of the same amount of protein in solution. In both cases, a third of the protein injected was fluorescently marked with Alexa Fluor 488. This staining allowed the in vivo non-invasive observation of the protein distribution in animal' eyes by fluorophotometry. While control eyes lost their fluorescent signal two weeks after administration, bevacizumab was still present two months after injection in eyes treated with the trojan microparticles. Two months post-dosing, eyes were enucleated and bevacizumab was detected by ELISA in vitreous, retina and choroid-RPE, but only in eyes treated with microspheres. All these results confirmed the high potential of the new methodology proposed for microencapsulation of active proteins system for vitreoretinal diseases.

Elsaid et al., (2016) [42] prepared "trojan" microparticles loaded with ranibizumab, an antibody fragment that binds all VEGF isoforms, having then antiangiogenic activity. They choose chitosan and $\mathrm{N}$-acetyl-cys-chitosan as biomaterials to prepare the nanoparticles. Although this biocompatible polymer is not yet accepted by regulatory agencies for intraocular administration, there is already a topical formulation in the market including $\mathrm{N}$-acetyl-cys-chitosan (Lacrimera ${ }^{\circledR}$ eye drops, Croma-Pharma) as lubricant for treatment of dry eye syndrome [43]. Chitosan nanoparticles can be prepared by different methods, being the ionotropic gelation the most employed. This method uses mild conditions and allows the formation of chitosan nanostructures alone or in association with other polymers such as hyaluronic acid, also used in this work. Interestingly, chitosan has demonstrated to have antiangiogenic activity itself, further improved by the presence of cysteine molecules in N-acetyl-cys-chitosan. This chitosan derivative offers, in addition, a better interaction with ranibizumab, and a more sustained release, due to the formation of disulfide bonds with thiol groups of the protein. Authors prepared ranibizumab-loaded chitosan (Mw $<400,000 \mathrm{~g} / \mathrm{mol}$ ) nanoparticles $(17-350 \mathrm{~nm})$ using plain chitosan, chitosan combined with hyaluronic acid or N-acetyl-Cys-Chitosan. After that, nanoparticles were included in PLGA (85:15; Mw $149,000 \mathrm{~g} / \mathrm{mol}$ ) microparticles prepared by $\mathrm{W} / \mathrm{O} / \mathrm{W}$ double emulsion and solvent extraction/evaporation method. Ramibizumab-loaded nanoparticles were suspended in water forming the inner aqueous phase. Furthermore, plain nanoparticles were also included at the same concentration in the outer aqueous phase. This astute strategy prevented the formation of a concentration gradient able to act as driving force for nanoparticles diffusion, jeopardizing their entrapment. The trojan microparticles created were evaluated in terms of $\mathrm{Z}$ potential and particle size using laser diffraction techniques and external morphology by SEM. Trojan microparticles resulted in the range of 3-6 $\mu \mathrm{m}$ for all batches, suitable for intravitreal injection. In the case of trojan systems prepared with $\mathrm{N}$-acetyl-Cys-chitosan significant lower size values were observed. According to authors, this phenomenon could be explained by an increased osmotic pressure inside the PLGA matrix promoted in presence of the chitosan derivative nanoparticles that might prevent the inward diffusion of water molecules during microparticles maduration. SEM pictures revealed the presence of chitosan nanoparticles on the trojan microparticles surface. No confocal studies were performed to confirm the nanoparticles distribution on the PLGA matrix. All trojan systems proposed resulted well tolerated according to mitochondrial activity of ARPE-19 cells (human retinal pigment epithelial cells) and HUVEC (human vascular endothelial cells) after 24 hours exposition to particles concentration from 0.5 to $10000 \mu \mathrm{g} / \mathrm{mL}$. The antiangiogenic activity of ranibizumab released from trojan microparticles was evaluated by the analysis of the inhibition of HUVEC migration, previously stimulated with VEGF. Native ranibizumab, used as control, reduced the cell migration 5-folds, similarly to the released protein from the different trojan prototypes proposed, ensuring that the manipulation of ranibizumab during nanoparticle and microparticles formation did not affect its activity. In the case of trojan microparticles containing $\mathrm{N}$-acetyl-cyschitosan, a slightly decrease of the VEGF-induced migration was even observed, probably due to the formation of secondary disulfide bonds between the polymer and the growth factor. Additionally, the antiangiogenic effect of chitosan, hyaluronic/chitosan loaded with ranibizumab or unloaded was measured by the capillary-like tube formation study. Sadly, no tube length inhibition studies were performed using the trojan systems. In any case, authors demonstrated that the systems created might be considered interesting platforms for the delivery of active anti-VEGF proteins after intravitreal administration.

\section{CONCLUSION}

Nowadays we are experiencing not only a raise of the life expectancy but also an increase of quality of life of aged population. As most of the vitreoretinal ocular diseases are age-related it is mandatory to offer more effective treatments that those currently in clinical practice. In this sense, the 
future of IODDS is promising. Among them, the use of nanoparticles-in-microparticle systems will have special relevance in the near future. The use of trojan microparticles will drastically improve the effectiveness at ocular level of already existent active compounds. Their utility to administrate bioengineered products is undeniable. Furthermore, it will help to reduce local side effects also appearing with small molecular weight drugs, such as the reduction of adverse effects in the lens associated to the intravitreal administration of corticoids anti-inflammatory drugs.

From a technological point of view, the use of trojan systems opens also the door to new co-delivery systems and might be of high utility to tailor the release profiles by combination of fast and slow releases and by reducing or increasing the burst effect according to the specific needs of each pathology/patient etc.

The formation and proper characterization of IODDS based on trojan systems is not exempt of problems. As pointed out in this review, it is important to ensure that the loaded dose of active compound remains in the nanoparticle structure during the microencapsulation process and ideally nanoparticles aggregation during this process might be avoided. Furthermore, as far as we have found in literature, no studies concerning the sterilization of trojan systems have been performed yet.

\section{LIST OF ABREVIATIONS}

Intraocular Drug Delivery System

IODDS

Nanoparticles -in-microparticles

NiMs

Retinal pigmented Epithelium

RPE

Poly-lactic-co-glycolic-acid

PLGA

Glial Cell Derived Factor

GDNF

Plasminogen Kringle

K5

Vascular Endothelial Growth Factor

VEGF

Acquired Immune Deficiency Syndrome

AIDS

Oligodeoxynucleotides-1

ODN-1

Water/Oil/Water Emulsion

$\mathrm{W} / \mathrm{O} / \mathrm{W}$

Water/Oli Emulsion

$\mathrm{W} / \mathrm{O}$

Solid/Water/Oil/Water Emulsion

$\mathrm{S} / \mathrm{W} / \mathrm{O} / \mathrm{W}$

Polyethylene glycol

PEG

Deoxyribonucleic acid

DNA

Polyethylene glycol - Poly Lactic Acid

PELA

Polyethyleneimine

PEI

Polyvinyl alcohol

PVA

Ultraviolet

UV

Transmission Electronic Microscopy

Scanning Electronic Microscopy
1,2-Dipalmitoil-sn-Glycerol-3-phosphocholine

Age Related Macular Degeneration

DPPC

Poly-lactic-acid

AMD

Carbon dioxide

PLA

Enzyme-Linked Immunosorbent Assay

$\mathrm{CO}_{2}$

Molecular weight

ELISA

Mw

Arising Retinal Pigment Epithelia

ARPE-19

Human Umbilical Vein Endothelial Cells

HUVEC

\section{CONFLICT OF INTEREST}

Authors do not have any conflict of interest

\section{FUNDING}

Research Group UCM 920415 (InnOftal). MINECO/AEI/FEDER, UE (MAT2017-83858-C2-1-R), MSCA-RISE-3DNEONET/734907 and the ISCII-FEDER RETICS (OFTARED) (RD16/0008/).

\section{REFERENCES}

[1] Kim, Y.C.; Chiang, B.; Wu, X.; Prausnitz, M.R., Ocular delivery of macromolecules. J Control Release, 2014, 190, 172-181.

[2] Herrero-Vanrell, R.; Vicario de la Torre, M.; Andrés-Guerrero, V.; Barbosa-Alfaro, D.; Molina-Martínez, I.T.; Bravo-Osuna, I., Nano and microtechnologies for ophthalmic administration, an overview. Journal of Drug Delivery Science and Technology, 2013, 23, (2), 75-102.

[3] Zhou, A.X.; Messenger, W.B.; Sargent, S.; Ambati, B.K., Safety of undiluted intracameral moxifloxacin without postoperative topical antibiotics in cataract surgery. International ophthalmology, 2016, 36, (4), 493-498.

[4] Yao, J.; Tucker, B.A.; Zhang, X.; ChecaCasalengua, P.; Herrero-Vanrell, R.; Young, M.J., Robust cell integration from co-transplantation of biodegradable MMP2-PLGA microspheres with retinal progenitor cells. Biomaterials, 2011, 32, (4), 1041-1050.

[5] Del Amo, E.M.; Rimpela, A.K.; Heikkinen, E.; Kari, O.K.; Ramsay, E.; Lajunen, T.; Schmitt, M.; Pelkonen, L.; Bhattacharya, M.; Richardson, D.; Subrizi, A.; Turunen, T.; Reinisalo, M.; Itkonen, J.; Toropainen, E.; Casteleijn, M.; Kidron, H.; Antopolsky, M.; Vellonen, K.S.; Ruponen, M.; Urtti, A., Pharmacokinetic aspects of retinal drug delivery. Progress in retinal and eye research, 2017, 57, 134-185.

[6] Herrero-Vanrell, R.; Bravo-Osuna, I.; AndresGuerrero, V.; Vicario-de-la-Torre, M.; Molina-Martinez, I.T., The potential of using biodegradable microspheres in retinal diseases and other intraocular pathologies. Prog Retin Eye Res, 2014, 42, 27-43. 
[7] Bravo-Osuna, I.; Andres-Guerrero, V.; Pastoriza Abal, P.; Molina-Martinez, I.T.; Herrero-Vanrell, R., Pharmaceutical microscale and nanoscale approaches for efficient treatment of ocular diseases. Drug Deliv Transl Res, 2016, 6, (6), 686-707.

[8] Bravo-Osuna, I.; Andres-Guerrero, V.; ArranzRomera, A.; Esteban-Perez, S.; Molina-Martinez, I.T.; Herrero-Vanrell, R., Microspheres as intraocular therapeutic tools in chronic diseases of the optic nerve and retina. Adv Drug Deliv Rev, 2018.

[9] Cardillo, J.A.; Souza-Filho, A.A.; Oliveira, A.G., Intravitreal Bioerudivel sustained-release triamcinolone microspheres system (RETAAC). Preliminary report of its potential usefulnes for the treatment of diabetic macular edema. Archivos de la Sociedad Espanola de Oftalmologia, 2006, 81, (12), 675-677, 679-681.

[10] Checa-Casalengua, P.; Jiang, C.; Bravo-Osuna, I.; Tucker, B.A.; Molina-Martinez, I.T.; Young, M.J.; HerreroVanrell, R., Retinal ganglion cells survival in a glaucoma model by GDNF/Vit E PLGA microspheres prepared according to a novel microencapsulation procedure. $J$ Control Release, 2011, 156, (1), 92-100.

[11] Garcia-Caballero, C.; Prieto-Calvo, E.; ChecaCasalengua, P.; Garcia-Martin, E.; Polo-Llorens, V.; GarciaFeijoo, J.; Molina-Martinez, I.T.; Bravo-Osuna, I.; HerreroVanrell, R., Six month delivery of GDNF from PLGA/vitamin E biodegradable microspheres after intravitreal injection in rabbits. Eur J Pharm Sci, 2017, 103, 19-26.

[12] Yoshida, T.; Gong, J.; Xu, Z.; Wei, Y.; Duh, E.J., Inhibition of pathological retinal angiogenesis by the integrin alphavbeta3 antagonist tetraiodothyroacetic acid (tetrac). Experimental eye research, 2012, 94, (1), 41-48.

[13] Park, K.; Chen, Y.; Hu, Y.; Mayo, A.S.; Kompella, U.B.; Longeras, R.; Ma, J.X., Nanoparticle-mediated expression of an angiogenic inhibitor ameliorates ischemiainduced retinal neovascularization and diabetes-induced retinal vascular leakage. Diabetes, 2009, 58, (8), 1902-1913.

[14] Crooke, S.T., Vitravene--another piece in the mosaic. Antisense \& nucleic acid drug development, 1998, 8, (4), vii-viii.

[15] Rayburn, E.R.; Zhang, R., Antisense, RNAi, and gene silencing strategies for therapy: mission possible or impossible? Drug Discov Today, 2008, 13, (11-12), 513-521. [16] Marano, R.J.; Toth, I.; Wimmer, N.; Brankov, M.; Rakoczy, P.E., Dendrimer delivery of an anti-VEGF oligonucleotide into the eye: a long-term study into inhibition of laser-induced $\mathrm{CNV}$, distribution, uptake and toxicity. Gene Ther, 2005, 12, (21), 1544-1550.

[17] Bhavsar, M.D.; Amiji, M.M., Gastrointestinal distribution and in vivo gene transfection studies with nanoparticles-in-microsphere oral system (NiMOS). $J$ Control Release, 2007, 119, (3), 339-348.

[18] Bhavsar, M.D.; Amiji, M.M., Oral IL-10 gene delivery in a microsphere-based formulation for local transfection and therapeutic efficacy in inflammatory bowel disease. Gene Ther, 2008, 15, (17), 1200-1209.
[19] Bhavsar, M.D.; Tiwari, S.B.; Amiji, M.M., Formulation optimization for the nanoparticles-inmicrosphere hybrid oral delivery system using factorial design. J Control Release, 2006, 110, (2), 422-430.

[20] Kriegel, C.; Amiji, M.M., Dual TNF-alpha/Cyclin D1 Gene Silencing With an Oral Polymeric Microparticle System as a Novel Strategy for the Treatment of Inflammatory Bowel Disease. Clin Transl Gastroenterol, 2011, 2, e2.

[21] Lee, Y.S.; Johnson, P.J.; Robbins, P.T.; Bridson, R.H., Production of nanoparticles-in-microparticles by a double emulsion method: a comprehensive study. Eur $J$ Pharm Biopharm, 2013, 83, (2), 168-173.

[22] Chen, Z.; Liu, D.; Wang, J.; Wu, L.; Li, W.; Chen, J.; Cai, B.C.; Cheng, H., Development of nanoparticles-inmicroparticles system for improved local retention after intra-articular injection. Drug Deliv, 2014, 21, (5), 342-350.

[23] Farris, E.; Brown, D.M.; Ramer-Tait, A.E.; Pannier, A.K., Chitosan-zein nano-in-microparticles capable of mediating in vivo transgene expression following oral delivery. J Control Release, 2017, 249, 150-161.

[24] Khan, I.U.; Serra, C.A.; Anton, N.; Er-Rafik, M.; Blanck, C.; Schmutz, M.; Kraus, I.; Messaddeq, N.; Sutter, C.; Anton, H.; Klymchenko, A.S.; Vandamme, T.F., Microfluidic conceived Trojan microcarriers for oral delivery of nanoparticles. Int J Pharm, 2015, 493, (1-2), 715.

[25] Guo, X.; Xia, T.; Wang, H.; Chen, F.; Cheng, R.; Luo, X.; Li, X., Electrosprayed microparticles with loaded pDNA-calcium phosphate nanoparticles to promote the regeneration of mature blood vessels. Pharm Res, 2014, 31, (4), 874-886.

[26] Elbaz, N.M.; Khalil, I.A.; Abd-Rabou, A.A.; ElSherbiny, I.M., Chitosan-based nano-in-microparticle carriers for enhanced oral delivery and anticancer activity of propolis. Int J Biol Macromol, 2016, 92, 254-269.

[27] Ozeki, T.; Akiyama, Y.; Takahashi, N.; Tagami, T.; Tanaka, T.; Fujii, M.; Okada, H., Development of a novel and customizable two-solution mixing type spray nozzle for one-step preparation of nanoparticle-containing microparticles. Biol Pharm Bull, 2012, 35, (11), 1926-1931.

[28] Deng, Y.; Mathaes, R.; Winter, G.; Engert, J., Encapsulation of antigen-loaded silica nanoparticles into microparticles for intradermal powder injection. Eur $J$ Pharm Sci, 2014, 63, 154-166.

[29] Tewes, F.; Ehrhardt, C.; Healy, A.M., Superparamagnetic iron oxide nanoparticles (SPIONs)loaded Trojan microparticles for targeted aerosol delivery to the lung. Eur J Pharm Biopharm, 2014, 86, (1), 98-104.

[30] Bakhtiary, Z.; Barar, J.; Aghanejad, A.; Saei, A.A.; Nemati, E.; Ezzati Nazhad Dolatabadi, J.; Omidi, Y., Microparticles containing erlotinib-loaded solid lipid nanoparticles for treatment of non-small cell lung cancer. Drug Dev Ind Pharm, 2017, 1-10.

[31] Schulze, J.; Kuhn, S.; Hendrikx, S.; SchulzSiegmund, M.; Polte, T.; Aigner, A., Spray-Dried Nanoparticle-in-Microparticle Delivery Systems (NiMDS) for Gene Delivery, Comprising Polyethylenimine (PEI)- 
Based Nanoparticles in a Poly(Vinyl Alcohol) Matrix. Small (Weinheim an der Bergstrasse, Germany), 2018, 14, (12), e1701810.

[32] Pinkerton, N.M.; Zhang, S.W.; Youngblood, R.L.; Gao, D.; Li, S.; Benson, B.R.; Anthony, J.; Stone, H.A.; Sinko, P.J.; Prud'homme, R.K., Gelation chemistries for the encapsulation of nanoparticles in composite gel microparticles for lung imaging and drug delivery. Biomacromolecules, 2014, 15, (1), 252-261.

[33] Garrait, G.; Beyssac, E.; Subirade, M., Development of a novel drug delivery system: chitosan nanoparticles entrapped in alginate microparticles. $J$ Microencapsul, 2014, 31, (4), 363-372.

[34] Gomez-Gaete, C.; Fattal, E.; Silva, L.; Besnard, M.; Tsapis, N., Dexamethasone acetate encapsulation into Trojan particles. J Control Release, 2008, 128, (1), 41-49.

[35] Algvere, P.; Bill, A., Drainage of microspheres and RBCs from the vitreous of aphakic and phakic eyes. Archives of ophthalmology (Chicago, Ill. : 1960), 1979, 97, (7), 1333-1336.

[36] Martens, T.F.; Vercauteren, D.; Forier, K.; Deschout, H.; Remaut, K.; Paesen, R.; Ameloot, M.; Engbersen, J.F.; Demeester, J.; De Smedt, S.C.; Braeckmans, $\mathrm{K}$., Measuring the intravitreal mobility of nanomedicines with single-particle tracking microscopy. Nanomedicine (Lond), 2013, 8, (12), 1955-1968.

[37] Koo, H.; Moon, H.; Han, H.; Na, J.H.; Huh, M.S.; Park, J.H.; Woo, S.J.; Park, K.H.; Kwon, I.C.; Kim, K.; Kim,
H., The movement of self-assembled amphiphilic polymeric nanoparticles in the vitreous and retina after intravitreal injection. Biomaterials, 2012, 33, (12), 3485-3493.

[38] Prow, T.W., Toxicity of nanomaterials to the eye. Wiley interdisciplinary reviews. Nanomedicine and nanobiotechnology, 2010, 2, (4), 317-333.

[39] Amrite, A.C.; Kompella, U.B., Size-dependent disposition of nanoparticles and microparticles following subconjunctival administration. The Journal of pharmacy and pharmacology, 2005, 57, (12), 1555-1563.

[40] Sherif, Z.; Pleyer, U., Corticosteroids in ophthalmology: past-present-future. Ophthalmologica. Journal international d'ophtalmologie. International journal of ophthalmology. Zeitschrift fur Augenheilkunde, 2002, 216, (5), 305-315.

[41] Yandrapu, S.K.; Upadhyay, A.K.; Petrash, J.M.; Kompella, U.B., Nanoparticles in porous microparticles prepared by supercritical infusion and pressure quench technology for sustained delivery of bevacizumab. Mol Pharm, 2013, 10, (12), 4676-4686.

[42] Elsaid, N.; Jackson, T.L.; Elsaid, Z.; Alqathama, A.; Somavarapu, S., PLGA Microparticles Entrapping ChitosanBased Nanoparticles for the Ocular Delivery of Ranibizumab. Mol Pharm, 2016, 13, (9), 2923-2940.

[43] Messina, M.; Dua, H.S., Early results on the use of chitosan-N-acetylcysteine $(\operatorname{Lacrimera}((\mathrm{R})))$ in the management of dry eye disease of varied etiology. International ophthalmology, 2018. 